\title{
A liga de meditação e saúde na educação, prevenção e terapêutica de profissionais de saúde e pacientes
}

\author{
The league of meditation and health in the education. prevention and therapeutics \\ of health professionals and patients
}

Marcela Usberti Gutierre', Robson Campos Gutierre², José Antonio Esper Curiati ${ }^{3}$

Gutierre MU, Gutierre RC, Curiati JAE. A liga de meditação e saúde na educação, prevenção e terapêutica de profissionais de saúde e pacientes / The league of meditation and health in the education. prevention and therapeutics of health professionals and patients. Rev Med (São Paulo). 2019 mar.-abr.;98(2):152-4.

RESUMO: O presente trabalho visa relatar as experiências associadas à Liga de Meditação e Saúde (LIMEDS), na Faculdade de Medicina da USP, desde sua fundação, em março de 2018, até o presente momento. Como Liga Acadêmica, o projeto exerce papel fundamental nas esferas de ensino, pesquisa e extensão, avançando no sentido de contribuir para a formação profissional no campo da saúde e, por meio de suas atividades teórico-práticas e assistenciais, atuar na prevenção e terapêutica de pacientes e profissionais de saúde. Em resposta à expansão das práticas meditativas no Brasil e no mundo, incluindo sua valorização em meios científicos, a LIMEDS assume o compromisso no combate e prevenção aos distúrbios psicossomáticos, no desenvolvimento da paz, compaixão e empatia, bem como na reorientação de práticas profissionais que beneficiem a saúde pública. Depoimentos de alunos revelam grande satisfação com as experiências e mudanças positivas em sua saúde física e mental.

Descritores: Meditação; Saúde mental; Saúde do estudante; Prática profissional.

\begin{abstract}
The present work aims to report the experiences associated with the League of Meditation and Health (LIMEDS), at the Faculty of Medicine of USP, from its foundation in March 2018, to the present moment. As an Academic League, the project plays a fundamental role in the areas of teaching, research and extension, advancing in the sense of contributing to professional training in the field of health and, through its theoretical and practical activities and assistance, to act in the prevention and therapeutics of patients and health professionals. In response to the expansion of meditative practices in Brazil and in the world, including its valorization in scientific circles, LIMEDS is committed to combating and preventing psychosomatic disorders, in the development of peace, compassion and empathy, as well as in the reorientation of professional practices that benefit the public health. Student testimonials reveal great satisfaction with the experiences and positive changes in their physical and mental health.
\end{abstract}

Keywords: Meditation; Mental health; Student health; Professional practice.

1. Acadêmica do Curso de Medicina, Faculdade de Medicina FMUSP, Universidade de São Paulo USP. https://orcid.org/0000-00030338-7960. Email: marcela.gutierre@fm.usp.br.

2. Pesquisador do Departamento de Neurologia e Neurocirurgia, Escola Paulista de Medicina, Universidade Federal de São Paulo, UNIFESP.

3. Professor de Geriatria Clínica, Departamento de Clínica Médica, Faculdade de Medicina FMUSP, Universidade de São Paulo - USP Endereço de correspondência: Marcela Usberti Gutierre. Rua Oscar Freire, 1504, apto 122A. Bairro Pinheiros, São Paulo, SP, Brasil. CEP: 05409-010. E-mail: marcela.gutierre@fm.usp.br 
Gutierre MU, et al. A liga de meditação e saúde na educação, prevenção e terapêutica de profissionais.

\section{INTRODUÇÃO}

\section{Definição}

Segundo a própria definição de seu Regulamento Interno, a Liga Acadêmica é "uma entidade estudantil autônoma com o objetivo de aprofundar o trinômio ensino, pesquisa e extensão em uma área específica do campo médico, complementando a formação médica acadêmica"1. Dentre suas inúmeras finalidades, são enunciadas: complementarização, atualização e difusão de conhecimentos, além de interação entre a universidade e a comunidade, bem como assistência à saúde social. Tendo em vista tais princípios e outros que as regem, pode-se dizer que o desenvolvimento das Ligas deve ser norteado pelas atuais e futuras demandas de saúde pública, a fim de que se cumpra o propósito de aprimorar a competência do profissional, conforme as reais necessidades sociais.

\section{Práticas Integrativas e Complementares}

Analisando a recente incorporação massiva de diversas técnicas meditativas nos hábitos de vida dos brasileiros, incluindo a já aplicação de tais métodos nos centros de saúde e Unidades Básicas de Saúde (UBS)², seguindo a Política Nacional de Práticas Integrativas e Complementares (PNPIC) do Ministério da Saúde ${ }^{3}$, é indubitável que a meditação tornou-se potencial transformador da saúde em nossa sociedade. Tal fenômeno, em parte, deve-se à atual conscientização social de que saúde não somente abrange os aspectos fisiológicos dos seres humanos, mas também integra sua mente e sua espiritualidade. Grandes universidades em todo o mundo já criaram centros de estudos baseados nessa visão mais moderna, investindo na meditação como ciência e instrumento de saúde pública ${ }^{4,5}$. Nesse contexto, portanto, a necessidade de mobilização de estudantes e profissionais da área de saúde para o aprendizado da meditação é indubitável, sendo as Ligas Acadêmicas um potencial alicerce nessa formação.

\section{RELATO DA LIGA}

\section{Histórico}

A Liga de Meditação e Saúde foi fundada em 3 de março de 2018, por iniciativa da aluna da Faculdade de Medicina da USP (FMUSP), Marcela Usberti Gutierre, e do Professor Doutor José Antonio Esper Curiati, geriatra no Hospital das Clínicas da Universidade de São Paulo. Pensando nos crescentes índices de distúrbios psicossomáticos da população e nos significativos benefícios e riscos vinculados à prática de meditação, a Liga tem por propósito ser ferramenta de aprendizado da ciência associada às diversas técnicas meditativas, orientando sua vivência correta e incluindo suas indicações e contraindicações, além de promover o bem-estar mental, físico e espiritual tanto dos atuais e futuros profissionais de saúde, como da comunidade em geral.

\section{Atividades - Relevância profissional, preventiva e terapêutica}

As atividades da Liga são baseadas na pluralidade de grupos de pesquisa em meditação já existentes na USP, além de convidados de outras instituições, e consistem, basicamente, em aulas semanais (às terças-feiras, das $19 \mathrm{~h}$ às $20 \mathrm{~h} 30$ ), ministradas por professores doutores renomados na área e por importantes líderes de tradicionais escolas de meditação. Os temas trabalhados são variáveis, incluindo abordagens mais científicas - com exposição de artigos e descobertas significativas associadas à meditação - e também abordagens mais práticas, apresentando diferentes técnicas meditativas existentes, suas origens, objetivos e impactos no indivíduo. As aulas são realizadas na FMUSP e, em geral, compostas por um período teórico e outro prático, permitindo que o indivíduo tenha consciência do que ocorre no organismo ao praticar a técnica aprendida.

Meditar tem repercussões profundas no corpo humano, sendo uma prática milenar que possui comprovação científica como prevenção e terapia do estresse, da ansiedade, da depressão, da dor, de estados inflamatórios e como ajuda no tratamento ao estresse pós-traumático e em diversos distúrbios, como os alimentares. Fortalece, também, a saúde mental e física dos indivíduos em diversos aspectos, como a memória, o sono, o sistema imune e inúmeras funções orgânicas. Ademais, é capaz de estimular em seus praticantes o desenvolvimento da compaixão, do altruísmo e a capacidade de refletir sob diferentes pontos de vista ${ }^{6,7}$.

Considerando tais fatos, a meditação é uma ferramenta de enorme potencial para promoção de saúde e seu aprendizado não deve, de modo algum, ser restrito à comunidade médica, mas sim dominado por todos os profissionais da área de saúde. Pensando nisso, a Liga de Meditação e Saúde abre vagas a todos os cursos de graduação envolvidos nesse campo (medicina, enfermagem, fonoaudiologia, nutrição, farmácia, saúde pública, terapia ocupacional, psicologia, educação física, fisioterapia, radiologia, serviço social e odontologia) e também a indivíduos já graduados nessas áreas, de qualquer instituição. Para serem admitidos como membros da Liga, os interessados devem ser aprovados na prova de admissão, realizada com base em um curso introdutório, que acontece anualmente.

Além disso, visando o compromisso com a assistência à saúde pública, a Liga define que um de seus encontros mensais seja aberto à população em geral (o chamado Encontro Aberto), empregando este momento para o ensino de técnicas meditativas básicas, aplicáveis 
no dia-a-dia, e para a orientação sobre as verdades e mitos da meditação. Os profissionais e alunos da área de saúde vivenciam esta experiência com o público leigo. Essas aulas também são ministradas por professores doutores e/ ou líderes espirituais. Para participarem, os interessados devem apenas se inscrever em um formulário divulgado na página do Facebook (ou enviado por e-mail, aos que não possuem perfil na rede social). $\mathrm{O}$ Encontro é gratuito e oferece 60 vagas ao público.

$\mathrm{O}$ aprendizado e a vivência da meditação pelos estudantes e profissionais de saúde, especificamente, podem trazer contribuições fundamentais para a sua formação e também para a sua própria qualidade de vida. Além de servir como nova ferramenta para prevenção e promoção de saúde, a meditação pode fortalecer os estudantes e os profissionais da área em seu dia-a-dia, quando são, constante e intensamente, desafiados a enfrentar o sofrimento, a doença, a fragilidade, a pressão e as decepções do ambiente que os cerca, evitando os quadros característicos de estresse desses indivíduos.

\section{Depoimento}

Segundo a aluna de medicina da FMUSP Nicole Mary Garcia de Carvalho, que cursou a LIMEDS durante o ano de 2018,

\begin{abstract}
"A liga é uma ótima oportunidade de vivenciar na teoria e na prática diferentes técnicas meditativas, apresentadas sob uma perspectiva cientifica por profissionais experientes e altamente capacitados, sem deixar de lado o acolhimento com que o curso nos é apresentado. Em um ambiente descontraído e que permite a convivência com pessoas das mais diversas áreas de atuação, a liga promove a saúde e o autoconhecimento de seus membros e da comunidade como um todo. Cada encontro surpreende pela riqueza de temas, imergindo nessa área do conhecimento tão pouco explorada na graduação e de grande relevância na prática médica".
\end{abstract}

\section{CONCLUSÃO}

Portanto, a Liga de Meditação e Saúde tem ação imprescindível para a saúde pública, sendo instrumento acadêmico de formação profissional e também de promoção e aprimoramento da qualidade de vida da população. Devese não somente preconizar a prática da meditação, mas compreendê-la, estudá-la e vivenciá-la integralmente, para que, assim, seus benefícios possam ser verdadeiramente, concretizados.

Agradecimentos: Agradecemos a acadêmica de Terapia Ocupacional da Faculdade de Saúde Pública da Universidade de São Paulo, Bruna Roberta da Silva, pela colaboração às atividades desta Liga Acadêmica, no cargo de diretora.

\section{REFERÊNCIAS}

1. Faculdade de Medicina da Universidade de São Paulo, Departamento Científico. Regulamento interno das Ligas Acadêmicas. Disponível por email: dc@fm.usp.br.

2. Brasil. Ministério da Saúde. Ministério da Saúde inclui 10 novas práticas integrativas no SUS. Disponível em: http://portalms.saude.gov.br/noticias/agencia-saude/42737ministerio-da-saude-inclui-10-novas-praticas-integrativasno-sus.

3. Brasil. Ministério da Saúde. Secretaria de Atenção à Saúde. Departamento de Atenção Básica. Política Nacional de Práticas Integrativas e Complementares no SUS - PNPICSUS. Brasília; 2006. Disponível em: http://bvsms.saude.gov. br/bvs/publicacoes/pnpic.pdf.
4. Semel Instiute. UCLA Longevity Center. Available from: https://www.semel.ucla.edu/longevity

5. Osher Collaborative for Integrative Medicine. Available from: https://www.oshercollaborative.org/.

6. King BG1, Conklin QA2, Zanesco AP3, Saron CD4. Residential meditation retreats: their role in contemplative practice and significance for psychological research. Curr Opin Psychol. 2019;28:238-44. doi: 10.1016/j. copsyc.2018.12.021.

7. Khoury B1, Knäuper B2, Schlosser M3, Carrière K2, Chiesa A4. Effectiveness of traditional meditation retreats: A systematic review and meta-analysis. J Psychosom Res. 2017;92:16-25. doi: 10.1016/j.jpsychores.2016.11.006. 